\title{
Analytical Study for the Nonlinear Vibrations of Multiwalled Carbon Nanotubes using Homotopy Analysis Method
}

\author{
M. M. Khader ${ }^{1,2}$, N. H. Sweilam ${ }^{3}$, Z. I. EL-Sehrawy ${ }^{4}$ and S. A.Ghwail ${ }^{4, *}$ \\ ${ }^{1}$ Department of Mathematics and Statistics, College of Science, Al-Imam Mohammad Ibn Saud Islamic University (IMSIU), Riyadh: \\ 11566, Saudi Arabia \\ 2 Department of Mathematics, Faculty of Science, Benha University, Benha, Egypt \\ ${ }^{3}$ Department of Mathematics, Faculty of Science, Cairo University, Giza, Cairo, Egypt \\ ${ }^{4}$ Department of Mathematics, Faculty of Science, Suez Canal University, Ismailia, Egypt
}

Received: 24 Jul. 2013, Revised: 26 Oct. 2013, Accepted: 27 Oct. 2013

Published online: 1 Jul. 2014

\begin{abstract}
In this article, the homotopy analysis method (HAM) is implemented for obtaining semi-analytical solutions to the problem of the nonlinear vibrations of multiwalled carbon nanotubes embedded in an elastic medium. A multiple-beam model is utilized in which the governing equations of each layer are coupled with those of its adjacent ones via the Van der Waals inter layer forces. The amplitude-frequency curves for large-amplitude vibrations of single-walled, double-walled and triple-walled carbon nanotubes are obtained. The influence of changes in material constants of the surrounding elastic medium and the effect of changes in nanotube geometrical parameters on the vibration characteristics are studied by comparing the results with those from the previous work. Series solutions of the problem under consideration are developed by means of HAM and the recurrence relations are given explicitly. The obtained numerical results show the rapid convergence of the series constructed by the proposed method to the exact solution. Test problems have been considered to ensure that HAM is accurate and efficient compared with the Adomian decomposition method.
\end{abstract}

Keywords: Nonlinear vibration, Carbon nanotube, Homotopy analysis method.

\section{Introduction}

It is well known that most of the scientific phenomena are modeled by ordinary or partial differential equations. Analytical solutions of these equations may well describe the various phenomena in science and nature, such as vibrations, solitons and propagation with a finite speed. The homotopy analysis method is an analytical technique for solving nonlinear differential equations devised by Shi-Jun Liao in 1992 ([11]-[13]). This method has been successfully applied to solve many types of nonlinear problems in science and engineering by many authors ([1], [7]-[9]), and references therein. We aim in this work to effectively employ HAM to establish the semi analytical solutions for the proposed nonlinear equations ([3]-[5], [10], [18]). By the present method, numerical results can be obtained with using a few iterations [18]. Moreover, HAM contains the auxiliary parameter $\hbar$, which provides us with a simple way to adjust and control the convergence region of solution series [13]. Therefore, HAM handles linear and nonlinear problems without any assumption and restriction. Moreover, this technique does not require any discretization, linearization or small perturbations. With the rapid development of nano-technology, there appears an ever-increasing interest of scientists and researchers in this field of science. Nano-materials, because of their exceptional mechanical, physical and chemical properties have been the main topic of research in many scientific publications. Nowadays, they are used as the substantial parts of nano-electronics, nano-devices, and nano-composites. One of these materials attracted great attention due to its high mechanical strength is carbon nano-tube (CNT). CNTs were discovered by Iijima in 1991. In spite of being too small and having light weight, they have very large Young's modulus in axial direction (nearly 1TPa).

\footnotetext{
* Corresponding author e-mail: suzy.future@gmail.com
} 
Undoubtedly, CNTs have the eligibility to be the new and most popular nano-material of this early part of the $21 \mathrm{st}$ century. Since the vibration of CNTs are of considerable importance in a number of nano-mechanical devices such as oscillators, charge detectors, field emission devices and sensors. Many researchers have been so far devoted to the problem of the vibration of these nano-materials. However, most of the investigations conducted on the vibration of multiwalled carbon nano-tubes (MWNTs) have been restricted to the linear regime and fewer works were done on the nonlinear vibration of these materials. For more details about this topic, see ([6], [15], [16], [21]).

\section{Basic idea of HAM}

To show the basic idea of HAM [11], we consider the following differential equation

$$
F[u(t)]=0,
$$

where $F$ is a nonlinear operator, $u(t)$ is an unknown function. For simplicity, we ignore all boundary and initial conditions, which can be treated in the similar way.

\subsection{Zeroth-order deformation equation}

Liao, constructed the so-called zeroth-order deformation equation

$$
(1-q) L\left[\Phi(t ; q)-u_{0}(t)\right]=q \hbar F[\Phi(t ; q)],
$$

where $L$ is an auxiliary linear operator, $u_{0}(t)$ is an initial guess, $\hbar$ is an auxiliary parameter and $q \in[0,1]$ is the embedding parameter. Obviously, when $q=0$ and $q=1$, it holds, respectively

$$
\Phi(t ; 0)=u_{0}(t), \quad \Phi(t ; 1)=u(t) .
$$

Thus, as $q$ increasing from 0 to 1 , the solution $\Phi(t ; q)$ various from $u_{0}(t)$ to $u(t)$. Expanding $\Phi(t ; q)$ in Taylor series with respect to the embedding parameter $q$, one has

$$
\Phi(t ; q)=u_{0}(t)+\sum_{m=1}^{\infty} u_{m}(t) q^{m}
$$

where

$$
u_{m}(t)=\left.\frac{1}{m !} \frac{\partial^{m} \Phi(t ; q)}{\partial q^{m}}\right|_{q=0} .
$$

Assume that the auxiliary linear operator, the initial guess and the auxiliary parameter $\hbar$ are selected such that the series (4) is convergent at $q=1$, Then at $q=1$ and by (3), the series (4) becomes

$$
u(t)=u_{0}(t)+\sum_{m=1}^{\infty} u_{m}(t)
$$

\subsection{The mth-order deformation equation}

Define the vector

$$
\mathbf{u}_{n}(t)=\left[u_{0}(t), u_{1}(t), \ldots, u_{n}(t)\right]
$$

Differentiating equation (2) $m$ times with respect to the embedding parameter $q$, then setting $q=0$ and dividing them by $m$ !, finally using (5), we have the so-called $m$ thorder deformation equations

$$
L\left[u_{m}(t)-\delta_{m} u_{m-1}(t)\right]=\hbar \Re_{m}\left(\mathbf{u}_{m-1}\right),
$$

where

$$
\mathfrak{R}_{m}\left(\mathbf{u}_{m-1}\right)=\left.\frac{1}{(m-1) !} \frac{\partial^{m-1} N[\Phi(t ; q)]}{\partial q^{m-1}}\right|_{q=0}
$$

and

$$
\delta_{m}= \begin{cases}0, & m \leq 1 \\ 1, & m>1\end{cases}
$$

\section{Implementation of HAM}

In this section, we apply HAM to obtain the approximate solution to the problem of the nonlinear vibrations of CNTs.

\subsection{Case 1:Nonlinear vibration of the SWNT}

Consider the SWNT of length $l$, Young's modulus $E$, density $\rho$, cross-sectional area $A$, and cross-sectional inertia moment $I$, embedded in an elastic medium with material constant $k$. The nonlinear vibration equation for this CNT is in the following form [2]

$$
\frac{d^{2} W}{d t^{2}}+\left(\frac{\pi^{4} E I}{\rho A l^{4}}+\frac{k}{\rho A}\right) W+\frac{\pi^{4} E}{4 \rho l^{4}} W^{3}=0,
$$

under the transformations

$r=\sqrt{\frac{I}{A}}, \quad x=\frac{W}{r}, w_{l}=\frac{\pi^{2}}{l^{2}} \sqrt{\frac{E I}{\rho A}}, w_{k}=\sqrt{\frac{k}{\rho A}}, \quad \tau=\omega t$, the above equation can be transformed to the following dimensionless nonlinear vibration equation

$$
\omega^{2} \frac{d^{2} x}{d \tau^{2}}+w_{b}^{2} x+\alpha w_{l}^{2} x^{3}=0
$$

in which $\alpha=0.25$ and $w_{b}=\sqrt{w_{l}^{2}+w_{k}^{2}}$, is the linear, free vibration frequency. With the initial conditions

$$
x(0)=X, \quad \dot{x}(0)=0 .
$$

By means of homotopy analysis method, we choose the approximation solution

$$
x_{0}(\tau)=X \cos \left(\psi w_{b} \tau\right) .
$$


This initial approximation is a trial function and it is used to obtain more accurate approximate solution of Eq.(12). Here $\psi$, is the ratio of the nonlinear frequency $\omega$, to the linear frequency $w_{b}$, where $X$ is the maximum vibration amplitude. By means of HAM, we choose the linear operator

$$
€[\Phi(\tau ; q)]=\frac{\partial^{2} \Phi(\tau ; q)}{\partial \tau^{2}}+\Phi(\tau ; q),
$$

where the operator $€$ satisfies the relation $\mathrm{E}\left[c_{1} \cos (t)+c_{2} \sin (t)\right]=0$ for some arbitrary constants $c_{1}, c_{2}$, also we define the non-linear operator as

$$
N(\Phi(\tau ; q))=w^{2} \frac{\partial^{2} \Phi(\tau ; q)}{\partial \tau^{2}}+w_{b}^{2} \Phi+\alpha w_{l}^{2} \Phi^{3},
$$

using the above definitions, we construct the zeroth-order deformation equation as follows

$$
(1-q) \mathrm{E}[\Phi(\tau ; q)-x(\tau)]=q \hbar_{1} N[\Phi(\tau ; q)] .
$$

Obviously when $q=0$ and $q=1$, we obtain

$$
\Phi(\tau ; 0)=x_{0}(\tau), \quad \Phi(\tau ; 1)=x(t)
$$

Therefore, as the embedding parameters $q$ increase from 0 to $1, \Phi(\tau ; q)$, varies from the initial guess $x_{0}(\tau)$ to the solution $x(\tau)$. Expanding $\Phi(\tau ; q)$ in Taylor series with respect to $q$

$$
\Phi(\tau ; q)=x_{0}(\tau)+\sum_{m=1}^{\infty} x_{m}(\tau ; q) q^{m}
$$

where

$$
x_{m}(\tau ; q)=\left.\frac{1}{m !} \frac{\partial^{m} \Phi(\tau ; q)}{\partial q^{m}}\right|_{q=0} .
$$

The initial guess and the auxiliary parameters $\hbar_{1}$ are properly chosen, the above is convergent at $q=1$

$$
x(\tau)=x_{0}(\tau)+\sum_{m=1}^{\infty} x_{m}
$$

which must one of the solution of the original nonlinear equation now we define the vector $\mathbf{x}_{n}=\left[x_{0}(\tau), x_{1}(\tau), x_{2}(\tau), \ldots\right]$. The $m$ th-order deformation equation is

$$
€\left[x_{m}(\tau)-\delta_{m} x_{m-1}(\tau)\right]=\hbar R_{m}\left(\mathbf{x}_{m-1}(\tau)\right),
$$

with the initial conditions $x_{m}(0)=0$ where

$$
R_{m}\left(x_{m-1}\right)=w^{2} \frac{d^{2} x_{m-1}(\tau)}{d \tau^{2}}+w_{b}^{2} x_{m-1}+\alpha w_{l}^{2} x_{m-1}^{3}
$$

Now the solution of the $m$ th-order deformation equation becomes

$$
x_{m}(t)=\delta_{m} x_{m-1}(t)+\hbar \int_{0}^{t} \int_{0}^{t}\left(R_{m}\left(x_{m-1}\right)\right) d \tau d \tau .
$$

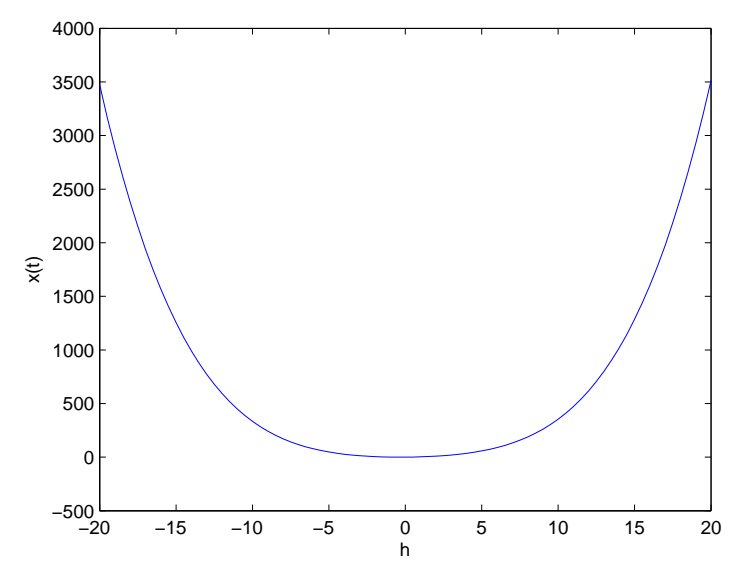

Figure 1. $\hbar$ curve of $x(\tau)$ for SWNT.

Substituting the initial approximation (14) into Eq.(12) results in the following residual

$$
\begin{aligned}
\overline{R_{0}}(\xi)= & \left(-X \Psi^{2}\left(w_{b}\right)^{2}+\left(w_{b}\right)^{2} X+0.75 \alpha\left(w_{l}\right)^{2} X^{3}\right) . \\
& \left.\cos \left(\Psi w_{b} \xi\right)+0.25\left(w_{l}\right)^{2}\right) X^{3} \cos \left(3 \Psi w_{b} \xi\right)
\end{aligned}
$$

In order to ensure that no secular terms appear in the next iteration, the coefficient of $\cos \left(\Psi w_{b} \xi\right)$ must vanish. Therefore $\Psi=\sqrt{1+\frac{3}{4} \alpha\left(w_{l} / w_{b}\right)^{2} X^{2}}$.

Now, by using the given initial condition (14), we can derive the first components of the solution. Then the approximate solution will take the following form

$$
\begin{aligned}
x_{m}(t) & =x_{0}(t)+x_{1}(t)+\ldots \\
& =\left[\frac{1}{20} \cos \left(\frac{t}{80}\right)-\hbar\left(\frac{2251}{3000}\right) \cos \left(\frac{t}{80}\right)\right. \\
& \left.+\frac{1}{18000} \cos ^{3}\left(\frac{t}{80}\right)-\ldots\right] .
\end{aligned}
$$

It is noted that our approximate solutions converge at $(-2 \leq \hbar \leq 2)$ (see Figure 1). The explicit, analytic expression given by Eq.(25) contains the auxiliary parameter $\hbar$, which gives the convergence region and rate of approximation for HAM. However, the errors can be further be reduced by calculating higher order approximations. This proves that HAM is a very useful method to get accurate analytic solutions to linear and strongly nonlinear differential equations. It must be noted that HAM used here gives the possibility for obtaining an analytical satisfactory solution for which the other techniques of calculation are more laborious and the results contain a great complexity. 


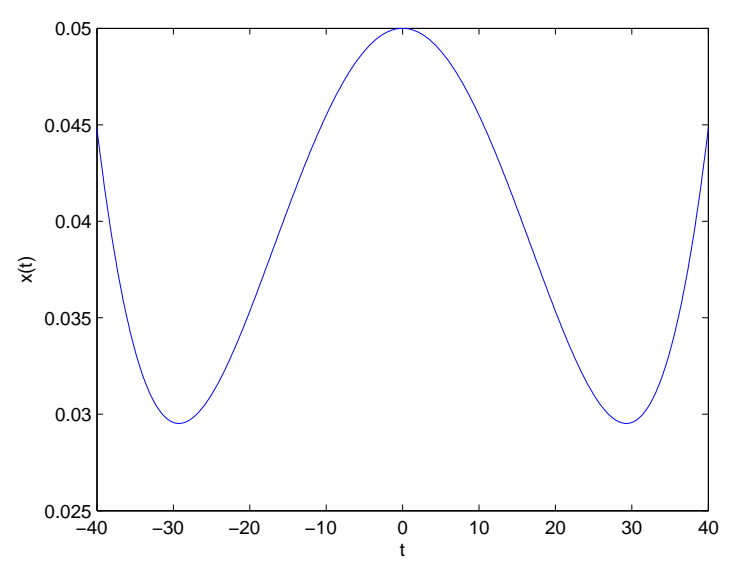

Figure 2. Approximate solution of SWNT for $\hbar=-0.5$.

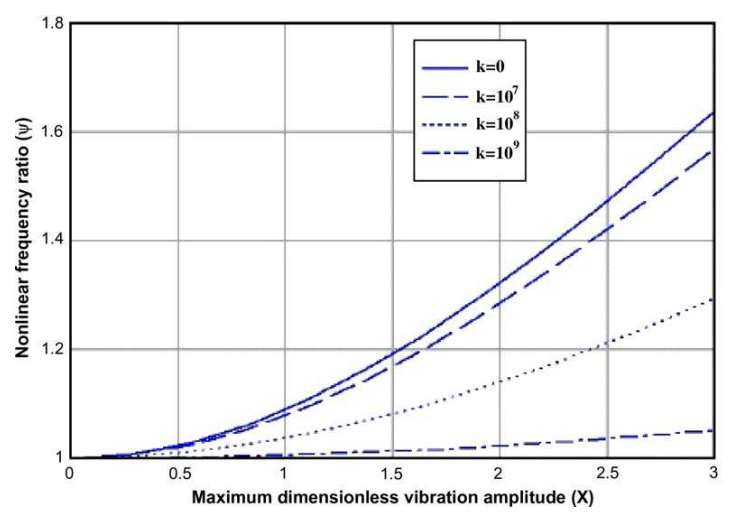

Figure 3. Effect of spring constant $k$ on nonlinear amplitudefrequency response curves of SWNT.

The amplitude-frequency response curves for the SWNT for different spring constants $k$ are shown in Figure 2. The material and geometric parameters taken here are $E=1.1 T P a, \rho=1300 \mathrm{~kg} / \mathrm{m}^{3}, \ell=45 \mathrm{~nm}$, the outer diameter $d_{1}=3 \mathrm{~nm}$ and the inner diameter $d_{0}=2.32 \mathrm{~nm}$. In this Figure 2, $\Psi$ is the ratio of nonlinear frequency to linear frequency as discussed earlier and $X$ is the maximum vibration amplitude. It can be seen that as the spring constant $k$ increases, the nonlinear frequencies tend to approach the linear ones especially when exceeds the value $10^{7} \mathrm{n} / \mathrm{m}^{2}$. It should be noted that from this figure is exactly the same as the figure obtained via incremental harmonic balance method (IHBM) [2] and Adomian decomposition method ([16], [17]).

\subsection{Case 2: Nonlinear vibration of the DWNT}

The nonlinear vibration governing equation for a DWNT is in the following form ([2], [21])

$$
\begin{aligned}
& \frac{d^{2} W_{1}}{d t^{2}}+\left(\frac{\pi^{4} E I_{1}}{\rho A_{1} l^{4}}+\frac{c_{1}}{\rho A_{1}}\right) W_{1}+\frac{\pi^{4} E}{4 \rho l^{4}} W_{1}^{3}-\frac{c_{1}}{\rho A_{1}} W_{2}=0 \\
& \frac{d^{2} W_{2}}{d t^{2}}+\left(\frac{\pi^{4} E I_{2}}{\rho A_{2} l^{4}}+\frac{c_{1}}{\rho A_{2}}+\frac{k}{\rho A_{2}}\right) W_{2}+\frac{\pi^{4} E}{4 \rho l^{4}} W_{2}^{3}-\frac{c_{1}}{\rho A_{2}} W_{1}=0,
\end{aligned}
$$

where $c_{1}$ is the coefficient of the Van der Waals force between the $(i$-th) tube and the $(i-1$ th) tube. By substituting the following dimensionless parameters

$$
\begin{aligned}
& r=\sqrt{\frac{I_{1}}{A_{1}}}, \quad x=\frac{W_{1}}{r}, \quad y=\frac{W_{2}}{r}, \\
& \omega_{l}=\frac{\pi^{2}}{l^{2}} \sqrt{\frac{E I_{1}}{\rho A_{1}}}, \quad \omega_{k}=\sqrt{\frac{k}{\rho A_{1}}}, \quad \omega_{c}=\sqrt{\frac{c}{\rho A_{1}}}, \\
& \tau=\omega t, \quad \beta=\frac{A_{1}}{A_{2}}, \quad \gamma=\frac{I_{1}}{I_{2}}, \quad \alpha=0.25 .
\end{aligned}
$$

Eqs.(26)-(27) can be transformed to the following dimensionless nonlinear system

$$
\begin{aligned}
& \left(\frac{\omega}{\omega_{l}}\right)^{2} \frac{d^{2} x}{d \tau^{2}}+B_{1} x+\alpha x^{3}-B_{2} y=0, \\
& \left(\frac{\omega}{\omega_{l}}\right)^{2} \frac{d^{2} y}{d \tau^{2}}+B_{3} y+\alpha y^{3}-B_{4} x=0,
\end{aligned}
$$

with $B_{1}$ to $B_{4}$ defined as

$$
\begin{gathered}
B_{1}=1+\left(\frac{\omega_{c}}{\omega_{l}}\right)^{2}, \quad B_{2}=\left(\frac{\omega_{c}}{\omega_{l}}\right)^{2} \\
B_{3}=\beta\left(\frac{1}{\gamma}+\left(\frac{\omega_{c}}{\omega_{l}}\right)^{2}+\left(\frac{\omega_{k}}{\omega_{l}}\right)^{2}\right), \quad B_{4}=\beta\left(\frac{\omega_{c}}{\omega_{l}}\right)^{2} .
\end{gathered}
$$

With the initial conditions

$$
x(0)=X_{1}, \quad y(0)=X_{2}, \quad \dot{x}(0)=\dot{y}(0)=0 .
$$

To solve system (29)-(30) by means of HAM, we choose the initial approximations

$$
x_{0}(\tau)=X_{1} \cos \left(\psi w_{b} \tau\right), \quad y_{0}(\tau)=X_{2} \cos \left(\psi w_{b} \tau\right) .
$$

We choose the linear operator

$$
€\left[\Phi_{i}(\tau ; q)\right]=\frac{\partial^{2} \Phi_{i}(\tau ; q)}{\partial \tau^{2}}+\Phi_{i}(\tau ; q), \quad i=1,2,
$$

where the operator $€$ satisfies the relation $€\left[c_{1} \cos (t)+c_{2} \sin (t)\right]=0$ for some arbitrary constants $c_{1}, c_{2}$, also we define the non-linear operators as

$$
\begin{aligned}
N_{1}\left(\Phi_{1}, \Phi_{2}\right)= & \left(\frac{\omega}{\omega_{l}}\right)^{2} \frac{\partial^{2} \Phi_{1}(\tau ; q)}{\partial \tau^{2}}+B_{1} \Phi_{1}(\tau ; q) \\
& +\alpha \Phi_{1}^{3}(\tau ; q)-B_{2} \Phi_{2}(\tau ; q),
\end{aligned}
$$




$$
\begin{aligned}
N_{2}\left(\Phi_{1}, \Phi_{2}\right)= & \left(\frac{\omega}{\omega_{l}}\right)^{2} \frac{\partial^{2} \Phi_{2}(\tau ; q)}{\partial \tau^{2}}+B_{3} \Phi_{2}(\tau ; q) \\
& +\alpha \Phi_{2}^{3}(\tau ; q)-B_{4} \Phi_{1}(\tau ; q) .
\end{aligned}
$$

Using the above definitions, we construct the zeroth-order deformation equations as follows

$$
\begin{aligned}
& (1-q) \mathrm{E}\left[\Phi_{1}(\tau ; q)-x_{0}(\tau)\right]=q \hbar_{1} N_{1}\left[\Phi_{1}, \Phi_{2}\right], \\
& (1-q) \mathrm{E}\left[\Phi_{2}(\tau ; q)-y_{0}(\tau)\right]=q \hbar_{2} N_{2}\left[\Phi_{1}, \Phi_{2}\right] .
\end{aligned}
$$

Obviously when $q=0$ and $q=1$, we obtain

$$
\begin{aligned}
& \Phi_{1}(\tau ; 0)=x_{0}(\tau), \quad \Phi_{2}(\tau ; 0)=y_{0}(\tau), \\
& \Phi_{1}(\tau ; 1)=x(\tau), \quad \Phi_{2}(\tau ; 1)=y(\tau) .
\end{aligned}
$$

Therefore, as the embedding parameters $q$ increase from 0 to $1, \Phi_{1}(\tau ; q)$ and $\Phi_{2}(\tau ; q)$ varies from the initial guess $x_{0}(\tau), y_{0}(\tau)$ to the solution $x(\tau), y(\tau)$, respectively. Expanding $\Phi_{1}(\tau ; q)$ and $\Phi_{2}(\tau ; q)$ in Taylor series with respect to $q$

$$
\Phi_{1}(\tau ; q)=\sum_{m=0}^{\infty} x_{m}(\tau) q^{m}, \quad \Phi_{2}(\tau ; q)=\sum_{m=0}^{\infty} y_{m}(\tau) q^{m} .
$$

where

$$
\begin{aligned}
& x_{m}(\tau ; q)=\left.\frac{1}{m !} \frac{\partial^{m} \Phi_{1}(\tau ; q)}{\partial q^{m}}\right|_{q=0}, \\
& y_{m}(\tau ; q)=\left.\frac{1}{m !} \frac{\partial^{m} \Phi_{2}(\tau ; q)}{\partial q^{m}}\right|_{q=0} .
\end{aligned}
$$

The initial guess and the auxiliary parameters $\hbar$ are properly chosen, the above series (39) are convergent at $q=1$

$$
x(\tau)=\sum_{m=0}^{\infty} x_{m}(\tau), \quad y(\tau)=\sum_{m=0}^{\infty} y_{m}(\tau),
$$

which must one of the solution of the original nonlinear equation, now we define the vectors

$\mathbf{x}_{n}=\left[x_{0}(\tau), x_{1}(\tau), x_{2}(\tau), \ldots\right], \quad \mathbf{y}_{n}=\left[y_{0}(\tau), y_{1}(\tau), y_{\tau}(\tau), \ldots\right]$.

The $m$ th-order deformation equations are

$$
\begin{aligned}
& €\left[x_{m}(\tau)-\delta_{m} x_{m-1}(\tau)\right]=\hbar_{1} R_{1, m}\left(\mathbf{x}_{m-1}, \mathbf{y}_{m-1}\right), \\
& €\left[y_{m}(\tau)-\delta_{m} y_{m-1}(\tau)\right]=\hbar_{2} R_{2, m}\left(\mathbf{x}_{m-1}, \mathbf{y}_{m-1}\right),
\end{aligned}
$$

with the initial conditions $x_{m}(0)=0$ and $y_{m}(0)=0$, where

$$
\begin{aligned}
R_{1, m}\left(\mathbf{x}_{m-1}, \mathbf{y}_{m-1}\right)= & \left(\frac{\omega}{\omega_{l}}\right)^{2} \frac{d^{2} x_{m-1}(\tau)}{d t^{2}}+B_{1} x_{m-1} \\
+ & \alpha x_{m-1}^{3}-B_{2} y_{m-1}, \\
R_{2, m}\left(\mathbf{x}_{m-1}, \mathbf{y}_{m-1}\right)= & \left(\frac{\omega}{\omega_{l}}\right)^{2} \frac{d^{2} y_{m-1}(\tau)}{d \tau^{2}}+B_{3} y_{m-1} \\
& +\alpha y_{m-1}^{3}-B_{4} x_{m-1} .
\end{aligned}
$$

Now, the solution of the $m$ th-order deformation equation (42) at $m \geq 1$ becomes

$$
\begin{aligned}
& x_{m}(\tau)=\delta_{m} x_{m-1}+\hbar_{1} \int_{0}^{t} \int_{0}^{t} R_{1, m}\left(\mathbf{x}_{m-1}, \mathbf{y}_{m-1}\right) d \tau d \tau, \\
& y_{m}(\tau)=\delta_{m} y_{m-1}+\hbar_{2} \int_{0}^{t} \int_{0}^{t} R_{2, m}\left(\mathbf{x}_{m-1}, \mathbf{y}_{m-1}\right) d \tau d \tau .
\end{aligned}
$$

In order to seek the periodic solution of Eqs.(29)-(30) substitute the initial approximations (32) into Eqs.(29)-(30) results in the following residuals

$$
\begin{gathered}
\overline{R_{1,0}}(\xi)=\left(-X_{1} \Psi^{2}\left(\omega_{b}\right)^{2}+\left(\omega_{l}\right)^{2} B_{1} X_{1}+0.75 \alpha\left(\omega_{l}\right)^{2} X_{1}^{3}\right. \\
\left.\left.-\omega_{l}^{2} B_{2} X_{2}\right) \cos \left(\Psi \omega_{b} \xi\right)+0.25 \alpha\left(\omega_{l}\right)^{2}\right) X_{1}^{3} \cos \left(3 \Psi \omega_{b} \xi\right), \\
\overline{R_{2,0}}(\xi)=\left(-X_{2} \Psi^{2}\left(\omega_{b}\right)^{2}+\left(\omega_{l}\right)^{2} B_{3} X_{2}+0.75 \alpha\left(\omega_{l}\right)^{2} X_{2}^{3}\right. \\
\left.\left.-\omega_{l}^{2} B_{4} X_{1}\right) \cos \left(\Psi \omega_{b} \xi\right)+0.25 \alpha\left(\omega_{l}\right)^{2}\right) X_{2}^{3} \cos \left(3 \Psi \omega_{b} \xi\right) .
\end{gathered}
$$

Here in $\Psi$, the ratio of the nonlinear frequency $\omega$ to the linear frequency $\omega_{b}$, is the unknown constant. Following the same approach as above and also eliminating the coefficient of $\cos \left(\psi \omega_{b} \xi\right)$ in the above system due to avoiding the secular terms, results in the following nonlinear system which can be easily solved using a simple mathematical algorithm such as Newton-Raphson technique

$$
\begin{aligned}
& -\left(\frac{\Psi}{\omega_{l}^{2}}\right) X_{1} \omega_{b}^{2}+B_{1} X_{1}+\frac{3}{4} \alpha X_{1}^{3}-B_{2} X_{2}=0, \\
& -\left(\frac{\Psi}{\omega_{l}^{2}}\right) X_{2} \omega_{b}^{2}+B_{3} X_{2}+\frac{3}{4} \alpha X_{2}^{3}-B_{4} X_{1}=0,
\end{aligned}
$$

to calculate the linear vibration frequencies for DWNT. We shall first substitute from (31) into Eqs.(29) and (30) without considering the nonlinear terms in Eqs.(29)-(30), so that

$$
\left(\begin{array}{cc}
\omega_{l}^{2}+\omega_{c}^{2}-\omega^{2} & -\omega_{c}^{2} \\
-\beta \omega_{c}^{2} & \beta\left(\frac{\omega_{l}^{2}}{\gamma}+\omega_{c}^{2}+\omega_{k}^{2}\right)-\omega^{2}
\end{array}\right)\left(\begin{array}{l}
X_{1} \\
X_{2}
\end{array}\right)=\left(\begin{array}{l}
0 \\
0
\end{array}\right),
$$

then by setting the determinant of the above matrix equal to zero, the frequency characteristic equation will be obtained. The fundamental linear vibration frequency of DWNT is the lowest root of the resulting equation.

Now, by using the given initial conditions (32) in the recurrence formula (45)-(46), we can obtain the first components of the solution, then the approximate solution will take the following form

$$
\begin{gathered}
x_{m}(\tau)=x_{0}(\tau)+x_{1}(\tau)+\ldots \\
\left.=\left(1-\frac{h_{1}}{6}\right) \cos (\tau)+\frac{1}{36} \cos ^{3}(\tau) \pm \ldots\right], \\
y_{m}(\tau)=y_{0}(\tau)+y_{1}(\tau)+\ldots \\
=\left[\left(1-\frac{19 h_{2}}{6} \cos (\tau)+\frac{1}{36} \operatorname{hboxcos}^{3}(\tau) \pm \ldots\right] .\right.
\end{gathered}
$$



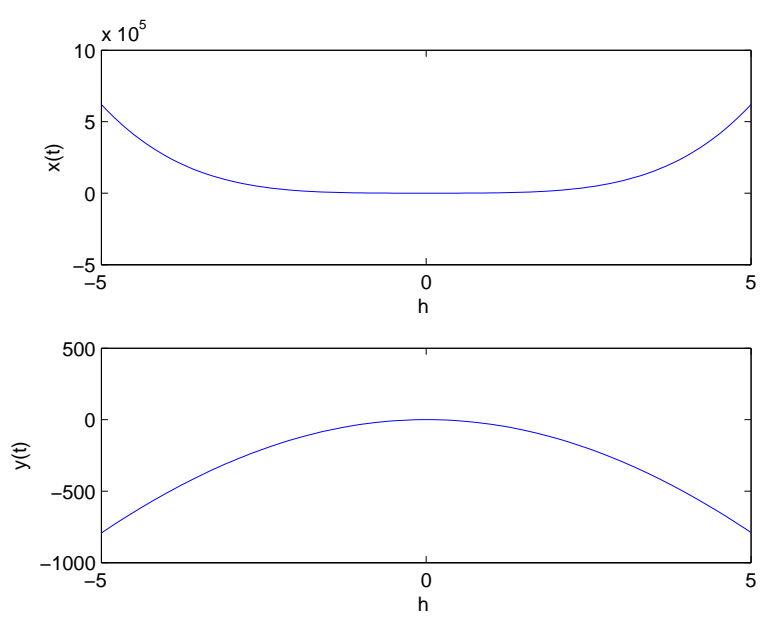

Figure 4. $h$ curve of $x(\tau)$ and $y(\tau)$ for DWNT.

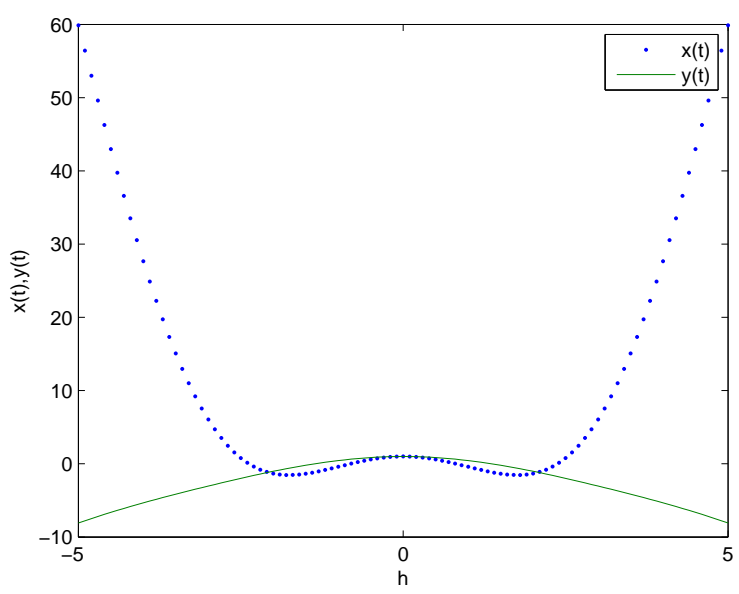

Figure 5. Approximate solution of DWNT for $\hbar=-1.5$.

It is noted that our approximate solutions converge at $(-2 \leq \hbar \leq 2)$ (see Figure 3 ). The explicit, analytic expression given by Eq.(45)-(46) contains the auxiliary parameter $\hbar$, which gives the convergence region $\hbar$ and rate of approximation for HAM. However, the errors can be further be reduced by calculating higher order approximations. This proves that HAM is a very useful method to get accurate analytic solutions to linear and strongly nonlinear differential equations. It must be noted that HAM used here gives the possibility for obtaining an analytical satisfactory solution for which the other techniques of calculation are more laborious and the results contain a great complexity.

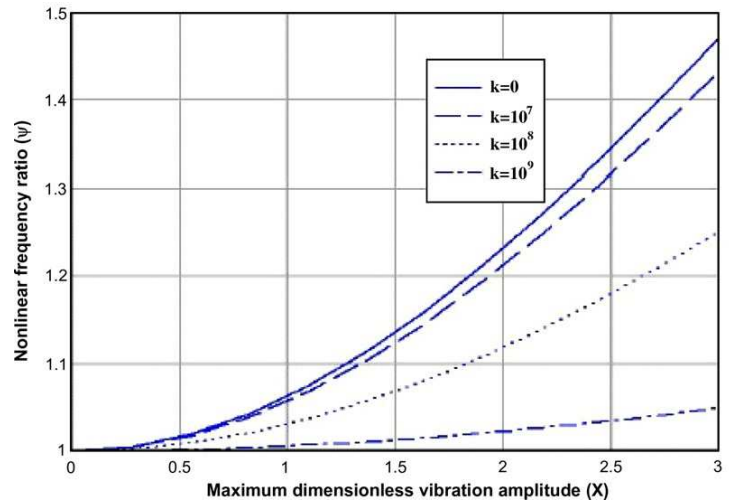

Figure 6. Effect of spring constant $k$ on nonlinear amplitudefrequency response curves of DWNT.

In Figure 4 shows the variation of the nonlinear amplitude-frequency response curves of DWNT against the maximum vibration amplitude for different spring constants $k$. The material and geometric parameters used to obtain this figure are, $E=1.1 \mathrm{TPa}, \rho=$ $1300 \mathrm{~kg} / \mathrm{m}^{3}, c=0.3 \times 10^{12} \mathrm{~N} / \mathrm{m}^{2}, l=45 \mathrm{~nm}$, $d_{0}=1.64 \mathrm{~nm}, d_{1}=2.32 \mathrm{~nm}$ and $d_{2}=3 \mathrm{~nm}$. It can be seen that the effect of spring constant on nonlinear vibration of DWNT is similar to that emerged in the case of SWNT (Figure 2) and this figure is exactly the same figure as that obtained via (IHBM) and Adomian decomposition method ([16], [17]).

\subsection{Case 3: Nonlinear vibration of a TWNT}

The nonlinear vibration governing equations for TWNTs are in the following form ([2], [19], [20])

$$
\frac{d^{2} W_{1}}{d t^{2}}+\left(\frac{\pi^{4} E I_{1}}{\rho A_{1} l^{4}}+\frac{c_{1}}{\rho A_{1}}\right) W_{1}+\frac{\pi^{4} E}{4 \rho l^{4}} W_{1}^{3}-\frac{c_{1}}{\rho A_{1}} W_{2}=0,
$$

$$
\begin{aligned}
& \frac{d^{2} W_{2}}{d t^{2}}\left.+\frac{\pi^{4} E I_{2}}{\rho A_{2} l^{4}}+\frac{c_{1}}{\rho A_{2}}+\frac{c_{2}}{\rho A_{2}}\right) W_{2} \\
&+\frac{\pi^{4} E}{4 \rho l^{4}} W_{2}^{3}-\frac{c_{1}}{\rho A_{2}} W_{1}-\frac{c_{2}}{\rho A_{2}} W_{3}=0 \\
& \frac{d^{2} W_{3}}{d t^{2}}+\left(\frac{\pi^{4} E I_{3}}{\rho A_{3} l^{4}}+\frac{c_{1}}{\rho A_{3}}+\frac{c_{2}}{\rho A_{3}}+\frac{k}{\rho A_{3}}\right) W_{3} \\
&+\frac{\pi^{4} E}{4 \rho l^{4}} W_{3}^{3}-\frac{c_{2}}{\rho A_{3}} W_{2}=0 .
\end{aligned}
$$


In a similar manner, introducing the following dimensionless parameters

$r=\sqrt{\frac{I_{1}}{A_{1}}}, \quad x=\frac{W_{1}}{r}, \quad y=\frac{W_{2}}{r}, \quad z=\frac{W_{3}}{r}$,

$\omega_{l}=\frac{\pi^{2}}{l^{2}} \sqrt{\frac{E I_{1}}{\rho A_{1}}}, \quad \omega_{k}=\sqrt{\frac{k}{\rho A_{1}}}, \omega_{c}=\sqrt{\frac{c}{\rho A_{1}}}, \tau=w t$,

$\beta=\frac{A_{1}}{A_{2}}, \quad \gamma=\frac{I_{1}}{I_{2}}, \quad \eta=\frac{A_{1}}{A_{3}}, \quad \zeta=\frac{I_{1}}{I_{3}}, \quad \alpha=0.25$

to the Eqs.(50)-(52) leads to the dimensionless nonlinear vibration equations as

$$
\begin{aligned}
& \omega^{2} \frac{d^{2} x}{d \tau^{2}}+\omega_{l}^{2} B_{1} x+\alpha \omega_{l}^{2} x^{3}-\omega_{l}^{2} B_{2} y=0 \\
& \omega^{2} \frac{d^{2} y}{d \tau^{2}}+\omega_{l}^{2} B_{3} y+\alpha \omega_{l}^{2} y^{3}-\omega_{l}^{2} \beta B_{2} x-\omega_{l}^{2} \beta B_{2} z=0
\end{aligned}
$$

$\omega^{2} \frac{d^{2} z}{d \tau^{2}}+\omega_{l}^{2} B_{4} z+\alpha \omega_{l}^{2} z^{3}-\omega_{l}^{2} \eta B_{2} y=0$,

with $B_{1}$ to $B_{4}$ defined as

$$
\begin{aligned}
& B_{1}=1+\left(\frac{\omega_{c}}{\omega_{l}}\right)^{2}, \quad B_{2}=\left(\frac{\omega_{c}}{\omega_{l}}\right)^{2}, \\
& B_{3}=\beta\left(\frac{1}{\gamma}+2\left(\frac{\omega_{c}}{\omega_{l}}\right)^{2}\right), \quad B_{4}=\eta\left(\frac{1}{\zeta}+2\left(\frac{\omega_{c}}{\omega_{l}}\right)^{2}+\left(\frac{\omega_{k}}{\omega_{l}}\right)^{2}\right) .
\end{aligned}
$$

With the initial conditions

$$
\begin{aligned}
& x(0)=X_{1}, \quad y(0)=X_{2}, \quad z(0)=X_{3}, \\
& \dot{x}(0)=\dot{y}(0)=\dot{z}(0)=0 .
\end{aligned}
$$

The initial approximations are selected by using the given initial conditions as

$$
\begin{aligned}
& x_{0}(\tau)=X_{1} \cos \left(\psi w_{b} t\right), \\
& y_{0}(\tau)=X_{2} \cos \left(\psi w_{b} t\right), \\
& z_{0}\left(\tau=X_{3} \cos \left(\psi w_{b} t\right) .\right.
\end{aligned}
$$

These initial approximations are trial functions and it used to obtain more accurate approximate solutions of Eqs.(53)(55), where

$$
\begin{gathered}
R_{1, m}\left(x_{m-1}, y_{m-1}, z_{m-1}\right)=\omega^{2} \frac{d^{2} x_{m-1}(\tau)}{d \tau^{2}}+\omega_{1}^{2} B_{1} x_{m-1} \\
+\alpha \omega_{1}^{2} x_{m-1}^{3}-\omega_{1}^{2} \beta B_{2} y_{m-1}
\end{gathered}
$$

$$
\begin{gathered}
R_{2, m}\left(x_{m-1}, y_{m-1}, z_{m-1}\right)=\omega^{2} \frac{d^{2} y_{m-1}(\tau)}{d \tau^{2}}+\omega_{1}^{2} B_{3} y_{m-1} \\
+\alpha y_{m-1}^{3}-\omega_{1}^{2} \beta B_{2} x_{m-1}-\omega_{1}^{2} \beta B_{2} z_{m-1}
\end{gathered}
$$

$$
\begin{gathered}
R_{3, m}\left(x_{m-1}, y_{m-1}, z_{m-1}\right)=\omega^{2} \frac{d^{2} z_{m-1}}{d \tau^{2}}+\omega_{l}^{2} B_{4} z_{m-1} \\
+\alpha \omega_{l}^{2} z_{m-1}^{3}-\omega_{l}^{2} \eta B_{2} y_{m-1}
\end{gathered}
$$

Now, the solution of the $m$ th-order deformation equations become $m \geq 1$ becomes

$$
\begin{aligned}
& x_{m}(\tau)=\delta_{m} x_{m-1}(\tau)+\hbar_{1} \int_{0}^{t} \int_{0}^{t} R_{1, m} d \tau d \tau, \\
& y_{m}(\tau)=\delta_{m} y_{m-1}(\tau)+\hbar_{2} \int_{0}^{t} \int_{0}^{t} R_{2, m} d \tau d \tau, \\
& z_{m}(\tau)=\delta_{m} z_{m-1}(\tau)+\hbar_{3} \int_{0}^{t} \int_{0}^{t} R_{3, m} d \tau d \tau .
\end{aligned}
$$

In order to seek the periodic solution of Eqs.(53)-(55) substitute the initial approximation (56) into Eqs.(53)-(55) results in the following residuals

$$
\begin{aligned}
\overline{R_{1,0}}(\xi) & =\left(-X_{1} \Psi^{2} \omega_{b}^{2}+\omega_{l}^{2} B_{1} X_{1}+0.75 \alpha \omega_{l}^{2} X_{1}^{3}\right. \\
& \left.\left.-\omega_{l}^{2} B_{2} X_{2}\right) \cos \left(\Psi \omega_{b} \xi\right)+0.25 \alpha \omega_{l}^{2}\right) X_{1}^{3} \cos \left(3 \Psi \omega_{b} \xi\right), \\
\overline{R_{2,0}}(\xi) & =\left(-x_{2} \Psi^{2} \omega_{b}^{2}+\omega_{l}^{2} B_{3} x_{2}+0.75 \alpha \omega_{l}^{2} x_{2}^{3}-\omega_{l}^{2} B B_{2} \beta x_{1}\right. \\
& \left.\left.-\omega_{l}^{2} B_{2} \beta x_{3}\right) \cos \left(\Psi \omega_{b} \xi\right)+0.25 \alpha \omega_{l}^{2}\right) x_{2}^{3} \cos \left(3 \Psi \omega_{b} \xi\right), \\
\overline{R_{3,0}}(\xi) & =\left(-x_{3} \Psi^{2} \omega_{b}^{2}+\omega_{l}^{2} B_{4} x_{3}+0.75 \alpha \omega_{l}^{2} x_{3}^{3}\right. \\
& \left.\left.-\omega_{l}^{2} B_{2} \eta x_{2}\right) \cos \left(\Psi \omega_{b} \xi\right)+0.25 \alpha \omega_{l}^{2}\right) x_{3}^{3} \cos \left(3 \Psi \omega_{b} \xi\right) .
\end{aligned}
$$

Here in $\Psi$, the ratio of the nonlinear frequency $\omega$ to the linear frequency $\omega_{b}$, is the unknown constant. Following the same approach as above and also eliminating the coefficient of $\cos \left(\psi \omega_{b} \xi\right)$ in the above system due to avoiding the secular terms, results in the following nonlinear system which can be easily solved using a simple mathematical algorithm such as Newton-Raphson technique

$$
\begin{aligned}
& -\left(\frac{\Psi}{\omega_{l}^{2}}\right) x_{1} \omega_{b}^{2}+B_{1} x_{1}+\frac{3}{4} \alpha x_{1}^{3}-B_{2} x_{2}=0 \\
& -\left(\frac{\Psi}{\omega_{l}^{2}}\right) x_{2} \omega_{b}^{2}+B_{3} x_{2}+\frac{3}{4} \alpha x_{2}^{3}-B_{2} \beta x_{1}-B_{2} \beta x_{3}=0, \\
& -\left(\frac{\Psi}{\omega_{l}^{2}}\right) x_{3} \omega_{b}^{2}+B_{4} x_{3}+\frac{3}{4} \alpha x_{3}^{3}-B_{2} \eta x_{2}=0
\end{aligned}
$$

to calculate the linear vibration frequencies for TWNT. We shall first substitute from (56) into Eqs.(53)-(55) without considering the nonlinear terms in Eqs.(53)-(55), so that

$$
\begin{aligned}
& \left(\begin{array}{ccc}
\omega_{l}^{2}+\omega_{c}^{2}-\omega^{2} & -\omega_{c}^{2} & 0 \\
-\beta \omega_{c}^{2} & \beta\left(\frac{\omega_{l}^{2}}{\gamma}+\omega_{c}^{2}+\omega_{k}^{2}\right)-\omega^{2} & -\beta \omega_{c}^{2} \\
0 & -\eta \omega_{c}^{2} & \eta\left(\frac{\omega_{l}^{2}}{\gamma}+\omega_{c}^{2}+\omega_{k}^{2}\right)-\omega^{2}
\end{array}\right) . \\
& \left(\begin{array}{l}
X_{1} \\
X_{2} \\
X_{3}
\end{array}\right)=\left(\begin{array}{l}
0 \\
0 \\
0
\end{array}\right) .
\end{aligned}
$$

Then by setting the determinant of the above matrix equal to zero, the frequency characteristic equation will be obtained. the fundamental linear vibration frequency of TWNT is the lowest root of the resulting equation.

Now, by using the given initial conditions (57), we can 

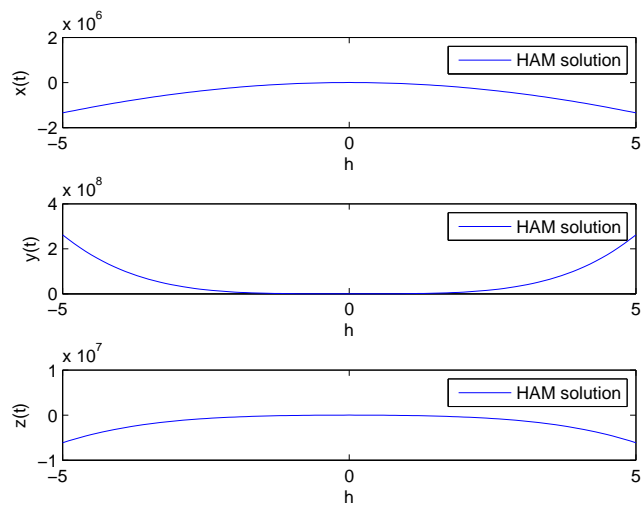

Figure 7. $h$ curve of $x(t), y(t)$ and $z(t)$ for TWNT.

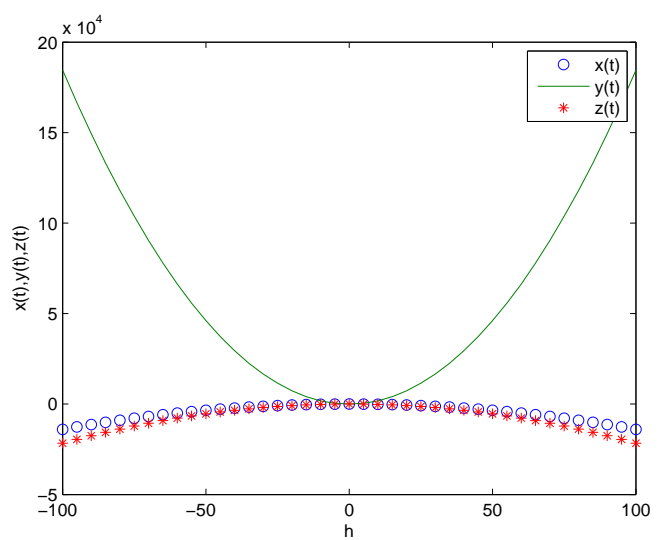

Figure 8. Approximate solution of TWNT for $\hbar=-1.25$.

obtain the first components of the solution. Then the approximate solution will take the following form

$$
\begin{aligned}
& x(\tau) \cong x_{0}(\tau)+x_{1}(\tau)=\left[1-\left(\frac{h_{1}}{6}\right) \cos (\tau)+\frac{1}{36} \cos ^{3}(\tau) \pm \ldots\right] \\
& y(\tau) \cong y_{0}(\tau)+y_{1}(\tau)=\left[1-\left(\frac{19 h_{2}}{6}\right) \cos (\tau)+\frac{1}{36} \cos ^{3}(\tau) \pm \ldots\right] \\
& z(\tau) \cong z_{0}(\tau)+z_{1}(\tau)=\left[\frac{-h}{36} \cos ^{3}(\tau)-\frac{5}{6} \cos (\tau) \pm \ldots\right] .
\end{aligned}
$$

It is noted that our approximate solutions converge at $(-2 \leq \hbar \leq 2)$. The explicit, analytic expression given by Eq.(65)-(67) contains the auxiliary parameter $\hbar$, which gives the convergence region and rate of approximation for HAM. However, the errors can be further be reduced by calculating higher order approximations. This proves that HAM is a very useful method to get accurate analytic solutions to linear and strongly nonlinear differential

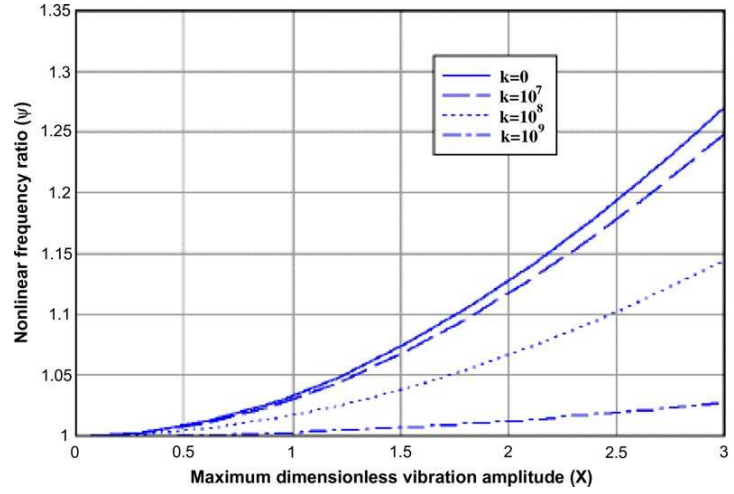

Figure 9. Effect of spring constant $k$ on nonlinear amplitudefrequency response curves of TWNT.

equations. It must be noted that HAM used here gives the possibility for obtaining an analytical satisfactory solution for which the other techniques of calculation are more laborious and the results contain a great complexity.

The variation of the nonlinear amplitude-frequency response curves of TWNT against the maximum vibration amplitude for different spring constants $k$ is also illustrated in figure 6. The material and geometric parameters used are

$c_{1}=c_{2}=0.3 \times 10^{12} \mathrm{~N} / \mathrm{m}^{2}, l=45 \mathrm{~nm}, d_{0}=0.96 \mathrm{~nm}$, $d_{1}=1.64 \mathrm{~nm}, d_{2}=2.32 \mathrm{~nm}$ and $d_{3}=3 \mathrm{~nm}$, clearly the same behavior as above is indefeasible in the case of DWNT. Due to convenience on calculating the nonlinear free vibration frequency $\omega$, the linear vibration frequencies $\omega_{b}(\mathrm{THz})$ of SWNT, DWNT and TWNT for all cases are listed in Table 1.

Table 1: The linear free vibration frequencies of SWNT, DWNT and TWNT in Figures 2, 4 and 6.

\begin{tabular}{|c|c|c|c|}
\hline$k\left(N / m^{2}\right)$ & SWNT: & DWNT: & DWNT: \\
\hline 0 & 0.128 & 0.116 & 0.111 \\
$10^{7}$ & 0.138 & 0.122 & 0.117 \\
$10^{8}$ & 0.209 & 0.170 & 0.156 \\
$10^{9}$ & 0.536 & 0.410 & 0.365 \\
\hline
\end{tabular}

\section{Conclusion and remarks}

In this paper, we implemented HAM to solve the problem of the nonlinear vibrations of multiwalled carbon nanotubes. The advantage of the method is that it does not require any discretization, linearization or small perturbations, leading to wide application in nonlinear problems. This method can be easily extended to the multiwalled CNTs with number of walls more than three. It may be concluded that this methodology is very 
powerful and efficient technique in finding exact solutions for wide classes of problems. It is also worth noting to point out that the advantage of this methodology shows a fast convergence of the solutions by means of the auxiliary parameter, $\hbar$. HAM is very easy applied to both differential equations and linear or nonlinear differential systems. The approximate solutions were almost identical to analytic solutions of the nonlinear evolution equations.

\section{References}

[1] S. Abbasbandy, Homotopy analysis method for heat radiation equations, International and Communication of Heat Mass Transfer, 34, 380-387 (2007).

[2] Y. M. Fu, J. W. Hong and X. Q. Wang, Analysis of nonlinear vibration for embedded carbon nanotubes, Journal of Sound and Vibration, 296, 746-756 (2006).

[3] I. Hashim, O. Abdulaziz and S. Momani, Homotopy analysis method for fractional IVPs, Communications in Nonlinear Science and Numerical Simulation, 14, 674-684 (2009).

[4] T. Hayat T, M. Khan M and S. Asghar, Homotopy analysis of MHD flows of an Oldroyd 8-constant fluid, Acta Mechanica, 168, 213-232 (2004).

[5] P. J. Hilton, An Introduction to Homotopy Theory, Cambridge University Press, Cambridge, (1953).

[6] Hossein Aminikah and Milad Hemmatnezhad, Nonlinear vibrations of multiwalled carbon nanotubes under various boundary conditions, International Journal of Differential Equations, 343576, 17 (2011).

[7] M. Inc, On exact solution of Laplace equation with Dirichlet and Neumann boundary conditions by the homotopy analysis method, Physics Letters A, 365, 412-415 (2007).

[8] H. Jafari and S. Seifi, Solving a system of nonlinear fractional partial differential equations using homotopy analysis method, Communications in Nonlinear Science and Numerical Simulation, 14, 1962-1969 (2009).

[9] M. M. Khader, Numerical solution for discontinued problems arising in nanotechnology using HAM, Nanotechnology and Advanced Materials An International Journal 2012 NSP, 1, 59-67 (2013).

[10] S. J. Liao, Beyond Perturbation: Introduction to the Homotopy Analysis Method, Champan \& Hall/CRC Press, Boca Raton, (2003).

[11] S. J. Liao, On the homotopy analysis method for nonlinear problems, Applied Mathematics and Computation, 147, 499-513 (2004).

[12] S. J. Liao, The Proposed Homotopy Analysis Technique for the Solution of Nonlinear Problems, PhD dissertation, Shanghai Jiao Tong University, (1992).

[13] S. J. Liao, Notes on the homotopy analysis method-Some definitions and theorems, Commun. Nonlinear Sci. Numer. Simulat., 14, 983-997 (2009).

[14] A. Molabahrami and F.Khani, The homotopy analysis method to solve the Burgers-Huxley equation. Nonlinear Anal. B: Real World Appl., 18-23 (2007) [doi:10.1016/j.nonrwa.10.014].

[15] Milad Hemmatnezhad and Hossein Aminikah, Applications of VIM to the nonlinear vibrations of multiwalled carbon nanotubes, The Open Applied Mathematics, 4, 18-23 (2010).
[16] L. Shuicai and Shi-Jun Liao, An analytic approach to solve multiple solutions of a strongly nonlinear problem, Applied Mathematics and Computation, 169, 854-865 (2005).

[17] N. H. Sweilam and M. M. Khader, Approximate solutions to the nonlinear vibrations of multiwalled carbon nanotubes using Adomian decomposition method, Applied Mathematics and Computation, 217, 495-505 (2010).

[18] N. H. Sweilam and M. M. Khader, Semi exact solutions for the bi-harmonic equation using homotopy analysis method, World Applied Sciences Journal, 13, 1-7 (2011).

[19] J. Yoon, C. Q. Ru and A. Mioduchowski, Vibration of an embedded multiwall carbon nanotube, Composites Science and Technology, 63, 1533-1542 (2003).

[20] J. Yoon, C. Q. Ru and A. Mioduchowski, Noncoaxial resonance of an isolated multiwall carbon nanotube, Physical Review B, 66, 233-402 (2002).

[21] Y. Zhang, G. Liu and X. Han, Transverse vibrations of double-walled carbon nanotubes under compressive axial load, Physics Letters A, 340, 258-266 (2005).

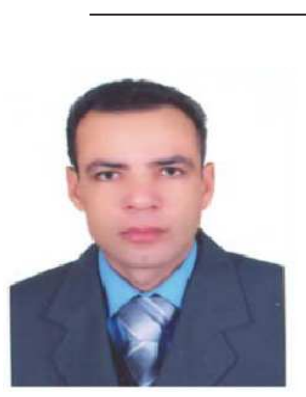

Mohamed M. Khader received the $\mathrm{PhD}$ degree in Mathematics Department -Faculty of Science-Benha University-Egypt (2009). His research interests are in the areas of Pure Mathematics including the mathematical methods and numerical techniques for solving FDEs. He has published research articles in reputed international journals of mathematical. $\mathrm{He}$ is referee of mathematical journals.

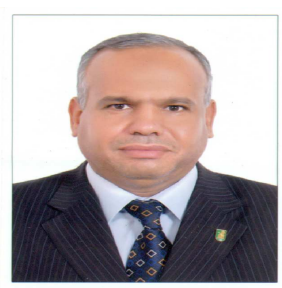

\section{Nasser H. Sweilam}

is professor of numerical analysis at the Department of Mathematics, Faculty of Science, Cairo University. He was a channel system Ph.D. student between Cairo University, Egypt, and TU-Munich, Germany. He received his Ph.D. in "Optimal Control of Variational Inequalities, the Dam Problem". He is the Head of the Department of Mathematics, Faculty of Science, Cairo University, science May 2012. He is referee and editor of several international journals, in the frame of pure and applied Mathematics. His main research interests are numerical analysis, optimal control of differential equations, fractional and variable order calculus, bio-informatics and cluster computing, ill-posed problems. 


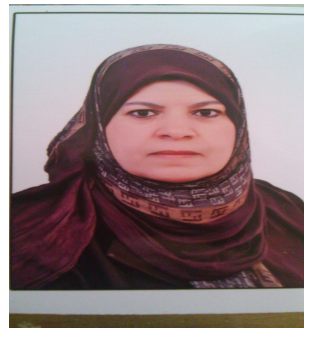

\section{Z. I. EL-Sehrawy}

received the $\mathrm{PhD}$ degree in Mathematics Department -Faculty of Science-Suez Canal University (Ismailia) -Egypt (1994). Her research interests are in the areas of Applied Mathematics in Computational Quantum Mechanics including the mathematical methods and numerical techniques for solving mathematical models.

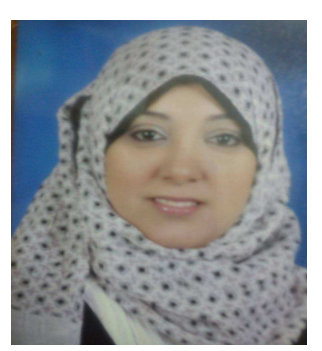

S. A. Ghwail

received the Msc degree in Mathematics Department, Suez Canal University (Ismailia) -(2011). Her research interests are in the areas of Applied Mathematics including the mathematical methods and numerical techniques for solving mathematical models in (Nano-technology, solitary waves,...). 